MRI of the shoulder by Michael B Zlatkin, Published by Lippincott, Williams and Wilkins; $2^{\text {nd }}$ Edition (2003); Hard bound; 306 pages; Rs. 6500/-

Choulder joints is one of the most extensively used joints of the Shuman body. Its anatomy is quite complex due to multiplicity of tendons and ligaments which support the osseo-cartilaginous elements. MRI is one modality which gives excellent multiplanar depiction of such complex anatomy. The author builds up the entire approach through chapters on physics of MRI, clinical aspects of shoulder pain, MRI imaging technique and ofcourse the interpretation of normal/abnormal images.

The book is specifically recommended for reference in all MRI centres, busy orthopaedic departments and sports medicine centres. Contributed by:

\section{Col MN Sree Ram}

Professor \& Head, Department of Radiodiagnosis and Imaging, Armed Forces Medical College, Pune-40.

\title{
Events Calendar
}

\section{$9^{\text {th }}-10^{\text {th }}$ October, 2004}

National Surgery Update 2004

Venue : Armed Forces Medical College

Pune-411 040

Contact person : Col GL Tiwari

Associate Professor

Department of Surgery

Armed Forces Medical College

Pune-411 040

Tel :- Mil : 6016

\section{$6^{\text {th }}-7^{\text {th }}$ November, 2004}

National anaesthesiology conference \& CME-2004

Venue : Armed Forces Medical College

Pune-411 040

Contact person : Col AK Bhargava

Professor \& Head

Department of Anaesthesiology \& Critical Care

Armed Forces Medical College

Pune-411 040

Tel :- Mil: 6036,6232,6274

Mobile No: 020-31025726

\section{APCON-2004}

Annual Conference of Pathologists and Microbiologists

20-25 November, 2004

Venue : Armed Forces Medical College, Pune-411 040

Phone : 020-2630 6024, 020-26306025

E-mail : apcon_2004@rediffmail.com

Website : www.geocites.com/apcon_2004

Corresp. to : Col S K Nema

Professor \& Head, Department of Pathology,

AFMC, Wanowri, Pune-411 040.

\section{$27^{\text {th }}-28^{\text {th }}$ November, 2004}

HOST-2004 'Haemorrhage in obstetrics' and 'Surgical techniques' Contact : Organising Secretary

Department of Obstetrics and Gynaecology

Armed Forces Medical College

Pune-411 040

Tel : 020-26306020, 020-26306021, Fax : 020-2687790

E-mail : host_2004@rediffmail.com 